\title{
Protean proteases: at the cutting edge of lung diseases
}

\author{
Clifford Taggart ${ }^{1}$, Marcus A. Mall ${ }^{2,3,4}$, Gilles Lalmanach ${ }^{5}$, Didier Cataldo ${ }^{6}$, \\ Andreas Ludwig ${ }^{7}$, Sabina Janciauskiene ${ }^{8}$, Nicole Heath ${ }^{3,4,9}$, Silke Meiners ${ }^{10}$, \\ Christopher M. Overall ${ }^{11}$, Carsten Schultz ${ }^{4,9}$, Boris Turk ${ }^{12}$ and \\ Keren S. Borensztajn ${ }^{13,14}$
}

\begin{abstract}
Affiliations: 'Airway Innate Immunity Research group (AiiR), Centre for Experimental Medicine, Queen's University Belfast, UK. ${ }^{2}$ Dept of Translational Pulmonology, University of Heidelberg, Heidelberg, Germany. ${ }^{3}$ Division of Pediatric Pulmonology \& Allergy and Cystic Fibrosis Center, Dept of Pediatrics, University of Heidelberg, Heidelberg, Germany. ${ }^{4}$ Translational Lung Research Center Heidelberg (TLRC), German Center for Lung Research (DZL), Heidelberg, Germany. ${ }^{5}$ INSERM UMR1100 Centre d'Etude des Pathologies Respiratoires (CEPR), Equipe: Mécanismes Protéolytiques dans l'Inflammation, Université François Rabelais, Tours, France. ${ }^{6}$ Laboratory of Tumors and Development and Dept of Respiratory Diseases, University of Liege, Liege, Belgium. ${ }^{7}$ Inflammation Pharmacology Research Group, Institute of Pharmacology and Toxicology, RWTH Aachen University, Aachen, Germany. ${ }^{8}$ Dept of Respiratory Medicine, a member of The German Center for Lung Research (DZL), Hannover Medical School, Hannover, Germany. ${ }^{9}$ European Molecular Biology Laboratory (EMBL), Heidelberg, Germany. ${ }^{10}$ Comprehensive Pneumology Center (CPC), University Hospital, LudwigMaximilians University, Helmholtz Zentrum München, Member of the German Center for Lung Research (DZL), Munich, Germany. ${ }^{11}$ Centre for Blood Research, Dept of Oral Biological and Medical Research University of British Columbia, Vancouver, BC, Canada. ${ }^{12}$ Dept of Biochemistry \& Molecular \& Structural Biology, J. Stefan Institute, Ljubljana, Slovenia. ${ }^{13}$ INSERM UMR_S933, Université Pierre et Marie Curie, Paris, France. ${ }^{14}$ INSERM UMR1152 Université Paris Diderot, Faculté de Médecine - site Bichat, Paris, France.
\end{abstract}

Correspondence: Keren S. Borensztajn, INSERM UMR_S933, Hôpital Trousseau, Université Pierre et Marie Curie, Paris, France. E-mail: keren.borensztajnđinserm.fr

@ERSpublications

Emergence of integrative protease biology offers new insights into the fundamental mechanisms of lung pathophysiology http://ow.ly/a7XH3077Muu

Cite this article as: Taggart C, Mall MA, Lalmanach G, et al. Protean proteases: at the cutting edge of lung diseases. Eur Respir J 2017; 49: 1501200 [https://doi.org/10.1183/13993003.01200-2015].

ABSTRACT Proteases were traditionally viewed as mere protein-degrading enzymes with a very restricted spectrum of substrates. A major expansion in protease research has uncovered a variety of novel substrates, and it is now evident that proteases are critical pleiotropic actors orchestrating pathophysiological processes. Recent findings evidenced that the net proteolytic activity also relies upon interconnections between different protease and protease inhibitor families in the protease web.

In this review, we provide an overview of these novel concepts with a particular focus on pulmonary pathophysiology. We describe the emerging roles of several protease families including cysteine and serine proteases.

The complexity of the protease web is exemplified in the light of multidimensional regulation of serine protease activity by matrix metalloproteases through cognate serine protease inhibitor processing. Finally, we will highlight how deregulated protease activity during pulmonary pathogenesis may be exploited for diagnosis/prognosis purposes, and utilised as a therapeutic tool using nanotechnologies.

Considering proteases as part of an integrative biology perspective may pave the way for the development of new therapeutic targets to treat pulmonary diseases related to intrinsic protease deregulation. 


\section{Introduction}

Proteases represent almost $2 \%$ of the human genome, comprising 565 members $[1,2]$. Based on their different catalytic mechanisms, five major classes of proteases are known in mammals, including serine, cysteine, metallo, aspartic, and threonine proteases, the three first families being the most widespread in humans [3]. Protease activity requires tight regulation (figure 1): elevated protease levels, actively contributing to disease progression, constitute a common feature shared by a broad range of pulmonary pathologies, including idiopathic pulmonary fibrosis (IPF), cystic fibrosis, emphysema or infection [4]. The underlying mechanisms of such observations have long remained elusive: indeed, proteases were traditionally viewed as mere protein-degrading enzymes with a restricted spectrum of substrates. During the past decade, a major expansion in protease research from different fields dramatically shifted this paradigm. The study of degradomics (the protease substrate repertoire) revealed that the nature of the dedicated substrates of proteases, and the biological effects triggered by their processing, are exceptionally diverse [5]. Proteases are now considered key components of regulatory mechanisms, through irreversible cleavage of substrates resulting in their activation, inactivation, or modulation of function. The serine proteases of the coagulation cascade constitute an archetypal example: beyond blood clot formation, these proteases contribute to a variety of pathophysiologies, including IPF, through the irreversible activation of their cellular receptors, the protease-activated receptors $[6,7]$. Knowledge of the cellular effects of other protease families, such as cysteine proteases or matrix-metalloproteases (MMPs), began to unfold only recently. Additionally, recent evidences show a regulation of protease activity through interconnection of protease cascades (the protease web).

In this review, these new concepts of integrative protease biology are discussed from the perspective of pulmonary pathophysiology. First, we highlight the striking role in lung pathogenesis of cysteine proteases, which were traditionally circumscribed to lysosomal degradation. We describe the recently discovered involvement of macrophage and neutrophil elastases, which (despite their names) actually belong to different clans, in cystic fibrosis. Defined localisation of protease activity is exemplified by the involvement of A disintegrin and metalloproteinase (ADAM) proteases in lung inflammation. Next, we focus on the main protease inhibitors, including serpins, to summarise the mechanisms underlying serpinopathies, and review recent evidence on serpin regulation by MMPs, thereby bolstering the concept of the protease web. These data are recapitulated in table 1. Finally, we highlight how deregulated protease activity during pulmonary pathogenesis may be exploited for diagnosis/prognosis purposes and utilised as a therapeutic tool using nanotechnologies.

\section{Unusual suspects: the emerging role of cathepsins, neutrophil and macrophage elastases, and ADAMs in lung diseases \\ Cysteinyl cathepsins: endolysosomal degradation and beyond}

It is only very recently that an unexpected role of cathepsins in lung diseases has emerged. Human cysteine cathepsins are a group of 11 papain-like lysosomal proteases including cathepsins B, H, L, C, X, F and O, and are ubiquitously expressed in most tissues, whereas cathepsins $\mathrm{V}, \mathrm{K}, \mathrm{S}$ and $\mathrm{W}$ have a more limited expression profile. Such diverse, tissue-specific expression suggests that their action is not limited solely to bulk protein degradation, but that they are also involved in the regulation of specific cellular functions. All of this allows cathepsins to regulate a broad variety of important physiological processes, including intracellular protein turnover, immune response, bone remodelling and prohormone processing $[8,9]$.

With respect to lung pathophysiology, cathepsins have a critical role in major histocompatibility class II-mediated antigen processing and presentation $[3,10,11]$. In addition, cathepsins, especially cathepsin K, have been found to have also major roles in the processing of TLR receptors $[10,12,13]$. In cystic fibrosis, levels of cathepsins B, L and S are increased in patient bronchoalveolar lavage fluid (BALF) [14], with bronchial epithelium acting a source of cathepsin S [15]. However, their relative contribution to extracellular proteolysis (as secreted proteases) versus intracellular proteolysis (as lysosomal proteases) remains to be determined, as does the balance between degradation and/or processing of specific substrates. Over the past decade, the role for cathepsins $\mathrm{K}$ and B in IPF has also emerged. In IPF patients, cathepsin $\mathrm{K}$ is increased in fibroblasts from fibrotic tissues. Unexpectedly, in the murine model of bleomycin-induced pulmonary fibrosis, cathepsin $\mathrm{K}$ deficiency associates with more severe lesions. Indeed,

Support statement: M.A. Mall was supported in parts by grants from the German Federal Ministry of Education and Research (82DZL00401 and 82DZL004A1). G. Lalmanach was supported by Région Centre-Val de Loire (France) (BPCO-Lyse project). S. Janciauskiene and N. Heath were supported by the German Center for Lung Research (DZL). B. Turk was supported by Slovene Research Agency (grant No P1-0140). K.S. Borensztajn was supported by grants from the Agence Nationale pour la Recherche ANR-14-CE15-0010-01, and Fondation pour la Recherche Médicale FRM AJE201121.

Conflict of interest: Disclosures can be found alongside this article at erj.ersjournals.com 


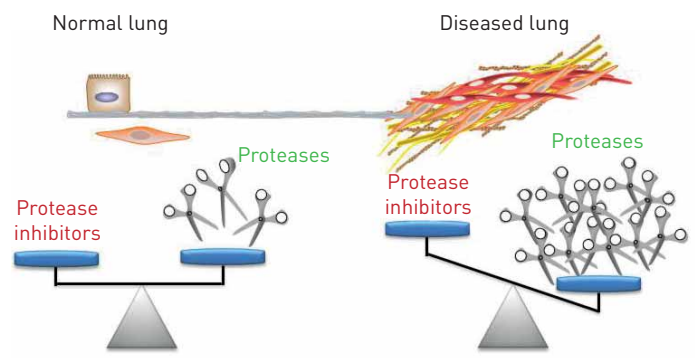

FIGURE 1 More-than-normal protease activity in lung pathogenesis. In the normal lung (left), proteases (represented by scissors) are involved in lung homeostasis and their activity is tightly regulated by (among others) cognate protease inhibitors. In a diseased lung (right), elevated protease activity, which can occur through increased protease expression and/or loss in cognate protease inhibitor, results into imbalanced protease activity.

cathepsin $\mathrm{K}$ has in fact a pivotal protective role in pulmonary homeostasis through collagen cleavage thereby contributing to the airway structural integrity [16-18]. Increased expression of cathepsin B was also observed in IPF patient-derived fibroblasts. Cathepsin B contributes to myofibroblast differentiation by triggering activation of the transforming growth factor (TGF)- $\beta 1$-driven canonical Smad 2-3 pathway [19]. Accordingly, cathepsin B inhibition and/or genetic silencing diminishes $\alpha$-smooth muscle actin expression and fibroblast differentiation.

From the above, the use of cathepsin pharmacological inhibitors seems an obvious therapeutical intervention. However, the off-targets and/or side-effects of cathepsin inhibitors remain to be fully elucidated. For instance, cathepsin S inhibitors efficiently reduce lung inflammation but can result in cartilage and bone degradation [20]. Cathepsin K inhibitors, developed for the treatment of osteoporosis, target cathepsin $\mathrm{K}$ collagenolytic activity and may impair collagen I turnover, thus favouring lung fibrosis [21]. A reversible cathepsin B inhibitor, VBY-376, demonstrated potent activity in a mouse model of $\mathrm{CCl}_{4}$-induced liver fibrosis [22]. Promising phase I trials for treatment of hepatic fibrosis have also been obtained with VBY-376 [23]. Since cathepsin B-driven responses are common in pulmonary and hepatic fibrosis, the use of VBY-376 may be appropriate for pulmonary fibrosis treatment.

Overall, it has emerged from the past decade that, beyond lysosomal degradation, cathepsins are crucial actors in pulmonary homeostasis and further research is warranted to better delineate their role in pulmonary pathophysiology.

\section{Neutrophil and macrophage elastases in cystic fibrosis: old dogs, new tricks}

An imbalance between neutrophil-derived proteases, including neutrophil elastase (NE), cathepsin G (CatG) and proteinase 3 (PR3), and extracellular inhibitors is considered an important pathogenic mechanism for a number of lung diseases [24-26]. It is assumed that unopposed CatG, NE and PR3 collectively cause severe lung damage and emphysema [27], further underscoring their importance in pathology (which has been the subject of a previous review [28]). A fourth elastase-related protease, NSP4, expressed in the lung, has been recently identified. Its proteolytic effects, controlled by natural serine protease inhibitors of the serpin type, are most likely limited to the intracellular and pericellular microenvironment of activated neutrophils. The natural substrates of NSP4, however, remain to be determined [29]. Recent studies on mouse NE revealed the existence of a new variant, created by the spontaneous conversion process of the single-chain form of NE into a specific two-chain form by autoprocessing. The existence of this two-chain NE form could adversely affect the protease-antiprotease balance in the lung during NE release, and may lead to the development of more efficient anti-elastase therapies [30].

Strikingly, recent studies in infants and preschool children with cystic fibrosis also identified the increased activity of $\mathrm{NE}$ as a key risk factor of the onset and progression of early bronchiectasis [31, 32]. In the context of chronic lung disease, $\mathrm{NE}$ is involved in a number of processes, including induction of interleukin (IL)-8 expression, increased mucin secretion, and inactivation of innate airway proteins and cell surface receptors, all of which, combined, identify NE as a pro-inflammatory molecule [33-40]. Similarly, in other chronic lung diseases, including chronic obstructive pulmonary disease (COPD), alpha-1 antitrypsin (A1AT) deficiency and bronchiectasis, NE is thought to contribute to disease pathogenesis [41-43].

However, it is in cystic fibrosis airways where the most definitive role regarding the deleterious effects of NE has been most clearly demonstrated, as shown by the AREST CF group [32]. The development of the $\beta \mathrm{ENaC}$-overexpressing ( $\beta \mathrm{ENaC}-\mathrm{Tg}$ ) mouse, featuring cystic fibrosis-like airway surface dehydration, phenocopies key characteristics of cystic fibrosis in patients, including airway inflammation, mucus hypersecretion and obstruction, bacterial infection and structural lung damage [44-46]. Recent studies in 
TABLE 1 Proteases with extracellular function associated with chronic lung diseases: mechanisms of action within the airway microenvironment, reporters and selected (commercially available) pharmacological inhibitors

\begin{tabular}{|c|c|c|c|}
\hline Protease & Mechanism of action & Reporter & Inhibitors \\
\hline \multicolumn{4}{|l|}{ Cysteine proteases } \\
\hline Cathepsin B & $\begin{array}{l}\text { Found in fibroblasts of IPF, contributes to myo-fibroblast } \\
\text { differentiation via TGF- } \beta 1 \text {, Smad 2-3 pathway [19] }\end{array}$ & $\begin{array}{c}\text { FRET reporter } \\
\text { (Hu, unpublished observations) }\end{array}$ & $\begin{array}{c}\text { VBY-376\# }[22] \\
\text { Leupeptin } \\
\text { E-64" }\end{array}$ \\
\hline Cathepsin K & Cleaves collagen, increased in fibroblasts of fibrotic tissue in IPF [16-18] & $\begin{array}{l}\text { FRET reporter (Subramanian, } \\
\text { unpublished observations) }\end{array}$ & $\begin{array}{l}\text { CTSK inhibitor II }{ }^{\#} \text { (Calbiochem } \\
\text { Cystatin } C^{\#}[98]\end{array}$ \\
\hline Cathepsin L & Increased in BALF of cystic fibrosis patients, along with cathepsin B and cathepsin K [16] & & $\begin{array}{l}\text { Pentapeptide amide } \\
\quad \text { RKLLW-NH2 }\end{array}$ \\
\hline Cathepsin S & Lysosomal protease, unlike other cathepsins remains active at neutral pH [99] & FRET reporter [100] & $\begin{array}{l}\text { Leupeptin } \\
\text { E-64ף }[15]\end{array}$ \\
\hline \multicolumn{4}{|l|}{ Serine proteases } \\
\hline Cathepsin-G & High affinity for nucleic acids, locates to NETs [28] & & Chymostatin $^{\#}$ \\
\hline NSP-4 & $\begin{array}{l}\text { Recently identified fourth neutrophil-derived protease lalong with } \\
\text { cathepsin G, NE and proteinase 3) substrate unknown [29] }\end{array}$ & & \\
\hline Neutrophil elastase & $\begin{array}{l}\text { Cleaves elastin, key risk factor for bronchiectasis in cystic fibrosis } \\
\qquad[30,31] \text {; induces IL-8, mucin secretion [33-40] }\end{array}$ & NEmo1, NEmo2 [47] & $\begin{array}{l}\text { Sivelestat } \\
\text { Elastase inhibitor Vף }\end{array}$ \\
\hline Proteinase-3 & Degrades elastin [101] & & \\
\hline \multicolumn{4}{|l|}{ Metalloproteases } \\
\hline ADAM-17 (TACE) & $\begin{array}{l}\text { Initiates endothelial permeabilty and leukocyte recruitment via } \\
\text { TNF generation }[42,43] \text {, involved in shedding TGF- } \alpha[48]\end{array}$ & & TAPI-1 [102] \\
\hline ADAM-10 & $\begin{array}{l}\text { Causes experimental emphysema [44], possible sheddase } \\
\text { for CD23 [45], involved in shedding TGF- } \alpha \text { [48] }\end{array}$ & & GI254023X $[103]$ \\
\hline ADAM-33 & Polymorphisms in ADAM-33 gene liked to asthma, COPD [46] & FRET probe $[104]$ & Anti-ADAM-33 antibody \\
\hline MMP-2 & $\begin{array}{l}\text { Cleaves type IV collagen, serum MMP-2 correlates with severity of pulmonary hypertension [105], } \\
\text { cleaves serpin B6 and alpha-1 inhibitor-3 [77] }\end{array}$ & & $\begin{array}{l}\text { GM6001\# }[106] \\
\text { Actinonin }\end{array}$ \\
\hline MMP-7 & Possible marker for IPF (serum, BALF) [107] & & $\begin{array}{l}\text { GM6001\# }[106] \\
\text { Actinonin }\end{array}$ \\
\hline MMP-8 & Cleaves serpin A1, releasing elastase activity in vivo [79] & & Actinonin \\
\hline MMP-9 (gelatinase B) & Cleaves type IV and V collagen, recruits leukocytes [108] & $\begin{array}{l}\text { FRET reporter (Halls, } \\
\text { unpublished observations) }\end{array}$ & $\begin{array}{l}\text { GM6001\# }[106] \\
\text { Actinonin }\end{array}$ \\
\hline MMP-10 & Possible marker for IPF (serum, BALF) [107] & & $\begin{array}{l}\text { GM6001\# }[106] \\
\text { Actinonin [107] }\end{array}$ \\
\hline MMP-12 (macrophage elastase) & $\begin{array}{l}\text { SNPs associated with lung function decline in COPD, asthma and } \\
\text { cystic fibrosis [48, 49] } \\
\text { Increased activity in smokers with COPD, asthma [36] and cystic } \\
\text { fibrosis [37], associated with lung function decline in cystic fibrosis [48] }\end{array}$ & LaRee1, LaRee5 [86] & $\begin{array}{l}\text { GM6001\# }[106] \\
\text { MMP408 } \\
\text { Actinonin } \\
\text { UK } 370106\end{array}$ \\
\hline MMP-14 & Processes SLPI [78] & & Anti-MMP-14 antibody \\
\hline
\end{tabular}


TABLE 1 Continued

Protease

Mechanism of action

Reporter

Inhibitors

Antiproteases

A1AT

Elafin (trappin-2)

SLP
Serpin, inhibits $N E$ cathepsin $G$, and proteinase 3 [64], deficiency causes emphysema and liver disease $[65,66]$, therapeutic potential for COPD [72]

Inhibits proteases, anti-microbial, modulates immune response [51, 52]

hibits proteases, anti-microbiat, modulates immune response [51,52], therapeutic potential for cystic fibrosis [74]

\#: competitive inhibition; ${ }^{\text {I: }}$ covalent inhibition. IPF: idiopathic pulmonary fibrosis; TGF: transforming growth factor; BALF: bronchoalveolar lavage fluid; NET: neutrophil extracellular trap; NSP-4: neutrophil serine protease-4; NE: neutrophil elastase; IL: interleukin; NEmo: neutrophil elastase monitor; ADAM: A disintegrin and metalloproteinase; TNF: tumour necrosis factor; COPD: chronic obstructive pulmonary disease; MMP: metalloprotease; SNP: single nucleotide polymorphism; LaRee: lavage reporter; SLPI: secretory leukocyte protease inhibitor; A1AT: alpha-1 antitrypsin. 
this cystic fibrosis model demonstrated that genetic deletion of NE reduces airway neutrophilia, mucin expression, goblet cell metaplasia and distal airspace enlargement without compromising anti-bacterial host defence in vivo [47]. In addition, whole-genome expression studies identified macrophage elastase (MMP-12) as a highly up-regulated gene in lungs from $\beta \mathrm{ENaC}-\mathrm{Tg}$ mice, and demonstrated that MMP-12 also contributes to structural lung damage in vivo [48]. The clinical relevance of this finding is supported by a genetic study showing that a functional single nucleotide polymorphism (rs2276109) in the MMP-12 promoter, previously associated with reduced lung function in COPD and asthma [49], was also associated with lung function decline in patients with cystic fibrosis [48]. More recent degradomic analysis of MMP-12 in vivo reveals its broad substrate variety. For instance, MMP-12 is potently anti-inflammatory in several in vivo models by cleaving and inactivating neutrophil chemokines [49], and terminating and complement factors, C4a and C5a [51, 52].

Taken together, these studies support important roles of elastases released from activated neutrophils and macrophages in the in vivo pathogenesis of multiple abnormalities in the cystic fibrosis lung, including inflammation, mucus hypersecretion and structural lung damage. These studies also demonstrate that the $\beta \mathrm{ENaC}-\mathrm{Tg}$ mouse will be a useful model for further studies of the roles of other emerging proteases including serine proteases, matrix metalloproteases and cysteine proteases $[15,25,51]$, and their respective inhibitors, in the complex in vivo pathogenesis of cystic fibrosis and potentially other chronic inflammatory lung diseases.

Localising the activity to the membrane: A disintegrin and metalloproteinase (ADAM proteases) The ADAM proteins constitute a major class of membrane-anchored multidomain proteinases that are responsible for the shedding of cell surface protein ectodomains, including the latent forms of growth factors, cytokines and receptors. As such, they are described as "signalling scissors". The ADAM family comprises $\sim 34$ members, 22 of which have been described in humans. Only 13 human ADAMs carry the zinc-binding motif (HExxHxxGxxH) required for proteolytic activity. Proteolytically active ADAMs can be regulated by induction of their gene transcription, by post-translational mechanisms upon stimulation with cytokines or bacterial toxins, or, in some cases, by cleavage of their ectodomain and subsequent release of a soluble form [52, 53]. Several members of proteolytically active ADAMs are involved in lung disease. ADAM17 is critical for generation of proinflammatory mediators, especially tumor necrosis factor (TNF)- $\alpha$. The shedding of soluble activated TNF- $\alpha$ subsequently stimulates a cascade of events causing endothelial permeability and leukocyte recruitment. The overexpression of ADAM10 in lung epithelium causes the development of experimental emphysema [54]. ADAM10 has been identified as potential sheddase for CD23 and the soluble form of this mediator enhances the production of IgE [55]. Polymorphisms in the ADAM33 gene are linked with asthma or COPD [56] and BALF levels of the soluble form of this protease correlate with the severity of the disease [57]. Additionally, ADAM10 and 17 are critically involved in the shedding of several growth factors, including TGF- $\alpha$, for example [58]. ADAMs are also implicated in host defence against infectious agents. ADAM10 functions as a receptor for Staphylococcus aureus toxin, and epithelial ADAM10 deficiency can prevent lethal S. aureus infection [59]. Finally, a key role has been proposed for ADAMs in airway remodelling associated with asthma [60]. Based on the above, it has been postulated that ADAM inhibition would decrease oedema formation, inflammatory cell recruitment, tissue remodelling, and reduction of $S$. aureus toxicity. Accordingly, the use of a selective ADAM17 inhibitor suppressed lipopolysaccharide-induced lung inflammation in mice [52]. From the above it is evident that ADAM proteases are implicated in acute and chronic inflammation processes but also in infection and regeneration. As inhibition of specific ADAMs might interfere with these processes, temporally and locally restricted and very specific inhibition strategies might be of interest for the treatment of lung disease.

\section{Proteases and natural serine protease inhibitors: the dangerous liaisons}

Besides an increase in protease expression and/or activation, deregulation of protease activity can arise from the loss of cognate protease inhibitors. Respiratory anti-proteases comprise serine protease inhibitors (serpins), tissue inhibitors of metalloproteinase, trappin-2/elafin, and secretory leukoprotease inhibitor (SLPI) [61]. These inhibitors not only neutralise protease activity, but also display anti-microbial and immune modulatory actions, which are highly relevant in chronic lung pathologies, such as in cystic fibrosis and COPD, where there is a combination of infection and exacerbated host responses [62, 63]. The clinical importance of protease inhibition is supported by studies demonstrating that inhibitor deficiencies result in structural lung damage. For instance, serpinopathies are a relatively newly defined group of disorders, where the underlying cause is the accumulation of misfolded protein, or lack of protein expression, in the affected tissue (figure 2). A1AT, a prototypical member of the serpin superfamily, is a specific inhibitor of NE, PR3 and catG [64]. Inherited A1AT deficiency, which causes familial emphysema and liver disease [65, 66], is a classic example of serpinopathy. A1AT deficiency is most often associated with the Z (Glu342Lys) mutation. Z-type A1AT forms oligomeric complexes; the intracellular retention of such complexes (gain-of-function defect) cause cellular damage $[67,68]$. Strikingly, cigarette smoke-mediated oxidation can 


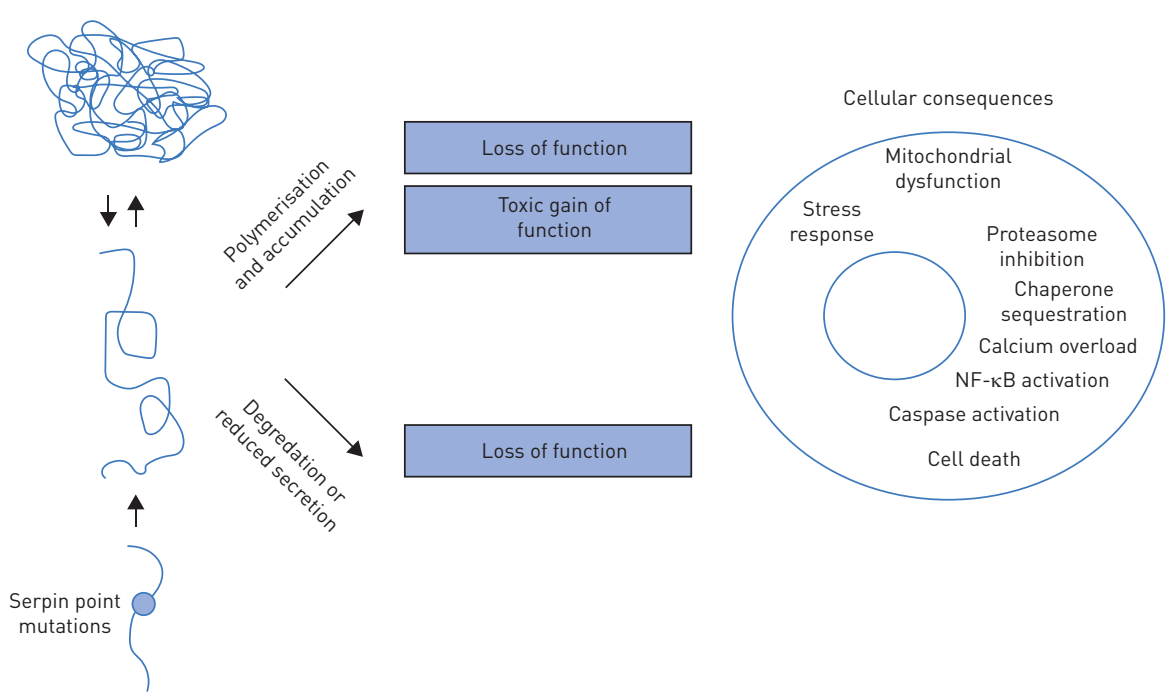

FIGURE 2 Mechanisms underlying serpinopathies. Point mutations in the gene encoding may result into misfolded protein and misfolded serpin polymerisation. Serpin polymers have no inhibitory functions towards serine proteases. Accumulation of serpin polymers has multiple cellular consequences. Other mutations can hinder protein expression. In either case, the loss of serpin results into uncontrolled proteolytic activity, thereby contributing to cell damage and the development of diseases.

enhance Z-A1AT polymerisation in human alveolar epithelial cells and induce endoplasmic reticulumstress and NF-кB-mediated pro-inflammatory cytokine (TNF- $\alpha$, IL- 6 and monocyte chemoattractant protein 1) production $[69,70]$.

Hence, anti-protease supplements may reduce the deleterious effects of unregulated NE activity. Accordingly, the therapeutic potential of natural endogenous inhibitors of proteases has gained interest over the past decade, and their newly appreciated functions point to extended therapeutic uses. Thus, human plasma-purified A1AT is used in infusion in patients with A1AT deficiency to delay the progression of emphysema. Administration of A1AT also triggers the anti-inflammatory pathways necessary for resolution of inflammation and prevention of tissue damage [71]. Inhalation therapy offers another opportunity for easier, more efficient delivery of A1AT directly to the lungs. Inhaled A1AT indeed significantly ameliorates cigarette smoke-induced emphysema in the mouse [72]. In patients with cystic fibrosis, A1AT inhalation reduces airway inflammation [73]. Similarly, aerosolised recombinant SLPI reduces elastase activity in healthy subjects and those with cystic fibrosis [74]. Altogether, these data indicate the potential value of recombinant natural inhibitors in the treatment of chronic lung disease.

Noteworthy, while cognate inhibitors are essential for protease activity regulation, recent evidence demonstrates that reciprocally, protease inhibitors are also protease substrates. Isotopic labels of cellular and secreted proteins in vitro and in vivo [75] revealed that serpins are major substrates of MMPs [76]. In other words, serine protease activity is regulated by MMPs through serpin processing. Accordingly, MMP-2 cleaves serpin B6 and alpha-1 inhibitor III [77], while MMP-14 processes SLPI [78]. Such a regulation of inhibitors through protease processing has significant consequences in pathophysiology. For instance, during lung inflammation, the cleavage of serpinA1 by MMP-8 releases elastase activity in vivo [79]. In arthritis, a protective role for macrophage MMP-12 has been demonstrated: among other mechanisms, MMP-12 induces antithrombin III degradation and increased thrombin activation [80]. In acute skin inflammation, MMP-2 cleaves serpin G1, also known as complement C1 inhibitor. These findings have unveiled a mechanism, referred to as "metallo-serpin switch", by which MMP-2 triggers complement cascade activation [75]. Altogether, these data revealed that proteases pervasively influence the activity of other proteases, either directly or by cleaving intermediate proteases or protease inhibitors.

\section{The protease web: is there a maze in the lung?}

As the aforementioned examples illustrate, proteases can no longer be thought of acting in isolation. Thus, proteases in the same or different families activate protease zymogens, inactivate proteases by autodegradation in trans, cleave and release protease substrate binding domains termed exosites (resulting in altered protease specificity and substrates), and, as exemplified above, cleave and inhibit protease inhibitors. By mapping these interactions of all reported biochemical validated cleavages in proteases and inhibitors uploaded to the data base MEROPS and TopFIND [81, 82], graph theory was used to bioinformatically map the protease interactions of proteases termed the protease web [79]. Remarkably, at 
the highly interconnected core of the protease web lie 255 highly connected proteases and inhibitors representing $50 \%$ of known proteases and inhibitors. Reachability analysis was performed, whereby the connections between proteases and inhibitors (paths) were mapped, and both the connections that are direct, i.e. 1:1, and indirect, i.e. further downstream reached by one or more intermediate connections. Thus, within this core, 158 of these proteins reached over $153(60 \%)$ of the protease and inhibitors of the core, spanning all five human protease classes. The protease web is highly robust. Removal of one or more nodes did not "break" this highly interconnected core, nor did removal of paths. It was not until $40 \%$ of paths were removed did the web connectivity break down. Similarly it required removal of more than six proteases or inhibitors to destabilise the interconnected core. The connectivity will be shown to further increase as more protease and inhibitor interactions are uploaded to MEROPS and TopFIND by individual investigations upon publication of their data. The important implication of these analyses is that therapy must be designed with great care as blockade of one protease will almost inevitably have knock on effects on other proteases and activity via connections in the protease web, and may explain the failure of previous protease inhibitor clinical trials [83-85].

It is noteworthy that the aforementioned findings have also recently emerged from other fields of research in addition to pulmonology. Since, as described earlier, several, if not all, of these actors have been shown to be involved in lung pathophysiology, it is tempting to speculate that these mechanisms are highly relevant in lung disease, and further research in this direction is eagerly awaited.

\section{Protease activity: a tool for diagnosis?}

Because elevated protease activity is associated with lung disease, it can be postulated that quantitative measurement of such an activity could serve as a surrogate marker for diagnosis/prognosis in lung diseases. A significant number of fluorescent probes have been developed to directly measure protease activities. In the late 1970s, short peptide-based molecules had already been developed to feature an intrinsic fluorescent entity.

While commercially available and still heavily in use, sophisticated fluorescent reporters, often based on Foerster Resonance Energy Transfer (FRET), have been developed in recent years. Frequently, two fluorophores are employed as FRET donor and acceptor. This provides the possibility to measure FRET with a more sensitive readout in a ratiometric fashion, mostly independent from the amount of reporter present. Recent examples are FRET sensors for MMP12 (LaRee series) and neutrophil elastase (NEmo series) [86, 87], which have revealed elevated MMP-12 and NE activity. While such probes work extremely well with isolated enzymes in the test tube, their application to biological samples is much more difficult. This is partly due to the fact that most proteases are counteracted by protease inhibitors, also present in mouse or patient samples. Further, the zymogen forms of proteases require cleavage for activation by other proteases and such activation seems to vary locally. For instance, when investigating MMP-12 activity from mouse bronchoalveolar lavage samples by the soluble reporter LaRee1, very little activity was observed. However, the lipidated reporter LaRee1, located to the outer cell membrane of bronchoalveolar lavage macrophages, reported strong activity. This demonstrates that the enzyme activity resides predominantly on cell surfaces and suggests that the lung lumen is much less exposed [86]. Similarly, NE seems to be mostly blocked in its proteolytic activity in samples from lung lumen, but the lipidated reporter was cleaved readily on the surface of activated neutrophils [87]. FRET-based reporters have now made their way to patient samples and revealed, for instance, elevated strong increase in MMP-12 and NE activity on the surface of airway neutrophils and macrophages from $\beta \mathrm{ENaC}-\mathrm{Tg}$ mice and patients with cystic fibrosis, respectively $[47,48]$. It is noteworthy that, very recently, different cathepsin-targeted imaging probes were developed and successfully used to monitor fibrotic disease progression in the murine model of bleomycin-induced pulmonary fibrosis and in IPF patients [88]. In the future, an extended array of reporters will help to characterise the protease activity landscape on cells isolated from patients and will hopefully assist physicians in the diagnosis of lung diseases, possibly predict disease course and aid the design of treatment strategies.

\section{Hijacking protease activity for therapeutics}

Dysregulation of MMP activity is a characteristic feature of organ fibrosis [89], such as that occurring in the lung [90] and malignant tumours [1,91]. Recent progress in the field of nanotechnology has focused on the use of MMP-directed nanoparticles as diagnostic and therapeutic or theranostic devices [92-95]. Compared to commonly used drug and imaging formulations, nano-scaled drug carriers offer additional advantages, such as increasing the drug circulation time, minimising drug degradation, and overcoming first-pass drug metabolism [96]. Mesoporous silica nanoparticles, engineered to release their incorporated chemotherapeutic drug upon gate-opening by MMP-2/9 cleavable linkers, have recently been developed [97]. Efficient drug release was shown to be strictly dependent on the presence of MMP-9 in human lung tumour cell lines; moreover, drug release was highly effective from mesoporous silica nanoparticles in 
mouse and human lung tumours. The successful application of therapeutic nanoparticles to ex vivo cultures of human lung tumours represents major progress in closing the translational gap in the development of novel MMP-targeted nanomedicines for lung tumour treatment. Thus, deregulation in protease activity in other lung diseases could be similarly hijacked for drug delivery and may represent a novel strategy for therapeutic intervention.

\section{Conclusion}

It is now clear that the traditional representation of proteases as mere extracellular matrix-degrading enzymes has shifted. The new paradigm allows a better understanding of how proteases are instrumental in the pathogenesis of a variety of chronic lung diseases. Protease deregulation during pathogenesis may occur through escape to its tight, spatio-temporal, self-limiting control. The wide variety of protease substrates adds a further complexity to this field. Indeed, the examples described in this review emphasise that the close interplay between proteases, substrates and inhibitors, which are interrelated in the protease interactome accounting for the net proteolytic activity in the lung, should be taken into consideration. As a result of the recent emergence of the aforementioned concepts, the understanding of the interplay and interdependency of these systems remains very scarce in the lung research field. Extrapolations of observations from different systems, together with translational approaches, combining degradomics data, murine models of lung disease and ex vivo observations from patients, should allow identification of an increasing number of instrumental proteases and protease inhibitors, in the perspective of integrative protease biology. Understanding in greater detail the complex molecular basis of regulation and function of protease activity will facilitate identification of new therapeutic targets as well as new diagnostic and prognostic tools for personalised medicine, and hopefully result in the development of new drugs to treat pulmonary diseases that result from inherent protease systems deregulation.

\section{References}

1 Lopez-Otin C, Overall CM. Protease degradomics: a new challenge for proteomics. Nat Rev Mol Cell Biol 2002; 3 : 509-519.

2 Puente XS, Sánchez LM, Overall CM, et al. Human and mouse proteases: a comparative genomic approach. Nat Rev Genet 2003; 4: 544-558.

3 Turk B, Turk D, Turk V. Protease signalling: the cutting edge. EMBO J 2012; 31: 1630-1643.

4 Tetley TD. New perspectives on basic mechanisms in lung disease. 6. Proteinase imbalance: its role in lung disease. Thorax 1993; 48: 560-565.

5 Doucet A, Overall CM. Protease proteomics: revealing protease in vivo functions using systems biology approaches. Mol Aspects Med 2008; 29: 339-358.

6 Borensztajn K, Peppelenbosch MP, Spek CA. Factor Xa: at the crossroads between coagulation and signaling in physiology and disease. Trends Mol Med 2008; 14: 429-440.

7 Mercer PF, Chambers RC. Coagulation and coagulation signalling in fibrosis. Biochim Biophys Acta 2013; 1832: 1018-1027.

8 Brix K, Dunkhorst A, Mayer, K, et al. Cysteine cathepsins: cellular roadmap to different functions. Biochimie 2008; 90: 194-207.

9 Turk V, Stoka V, Vasiljeva, O, et al. Cysteine cathepsins: from structure, function and regulation to new frontiers. Biochim Biophys Acta 2012; 1824: 68-88

10 Bird PI, Trapani JA, Villadangos JA, Endolysosomal proteases and their inhibitors in immunity. Nature reviews. Nat Rev Immunol 2009; 9: 871-882.

11 Zavasnik-Bergant T, Turk B. Cysteine proteases: destruction ability versus immunomodulation capacity in immune cells. Biol Chem 2007; 388: 1141-1149.

12 Fonovic UP, Jevnikar Z, Kos J. Cathepsin S generates soluble CX3CL1 (fractalkine) in vascular smooth muscle cells. Biol Chem 2013; 394: 1349-1352.

13 Repnik U, Starr AE, Overall CM, et al. Cysteine Cathepsins Activate ELR Chemokines and Inactivate Non-ELR Chemokines. J Biol Chem 2015; 290: 13800-13811.

14 Taggart CC, Greene CM, Smith SG, et al. Inactivation of human beta-defensins 2 and 3 by elastolytic cathepsins. Immunol 2003; 171: 931-937.

15 Weldon S, McNally P, McAuley DF, et al. miR-31 dysregulation in cystic fibrosis airways contributes to increased pulmonary cathepsin S production. Am J Respir Crit Care Med 2014; 190: 165-174.

16 Bühling F, Röcken C, Brasch F, et al. Pivotal role of cathepsin K in lung fibrosis. Am J Pathol 2004; 164: 2203-2216.

17 Srivastava M, Steinwede K, Kiviranta R, et al. Overexpression of cathepsin K in mice decreases collagen deposition and lung resistance in response to bleomycin-induced pulmonary fibrosis. Respir Res 2008; 9: 54.

18 Zhang D, Leung N, Weber E, et al. The effect of cathepsin K deficiency on airway development and TGF-betal degradation. Respir Res 2011; 12: 72.

19 Kasabova M, Joulin-Giet A, Lecaille F, et al. Regulation of TGF-beta1-driven differentiation of human lung fibroblasts: emerging roles of cathepsin B and cystatin C. J Biol Chem 2014; 289: 16239-16251.

20 Wilkinson RD, Williams R, Scott CJ, et al. Cathepsin S: therapeutic, diagnostic, and prognostic potential. Biol Chem 2015; 396: 867-882.

21 Bromme D, Lecaille F. Cathepsin K inhibitors for osteoporosis and potential off-target effects. Expert Opin Investig Drugs 2009; 18: 585-600.

22 Holsinger L, Coakley DF, Dener J, et al. Efficacy of a reversible cathepsin B inhibitor in a rodent model of liver fibrosis and human pharmacokinetic profile. Hepatology 2010; 52:1128A. 
Virobay/Adis Insight. Drug profile VBY 376. http://adisinsight.springer.com/drugs/800031124 Date last updated: April 18, 2016.

Cantin AM, Hartl D, Konstan MW, et al. Inflammation in cystic fibrosis lung disease: pathogenesis and therapy. J Cyst Fibros 2015; 14: 419-430.

Twigg MS, Brockbank S, Lowry P, et al. The role of serine proteases and antiproteases in the cystic fibrosis lung. Mediators Inflamm 2015; 2015: 293053.

Nichols DP, Chmiel JF. Inflammation and its genesis in cystic fibrosis. Pediatr Pulmonol 2015; 50: Suppl 40 S39-S56.

Guyot N, Wartelle J, Malleret L, et al. Unopposed cathepsin G, neutrophil elastase, and proteinase 3 cause severe lung damage and emphysema. Am J Pathol 2014; 184: 2197-2210.

Korkmaz B, Horwitz MS, Jenne DE, et al. Neutrophil elastase, proteinase 3, and cathepsin G as therapeutic targets in human diseases. Pharmacol Rev 2010; 62: 726-759.

Perera NC, Schilling O, Kittel H, et al. NSP4, an elastase-related protease in human neutrophils with arginine specificity. Proc Natl Acad Sci USA 2012; 109: 6229-6234.

Dau T, Sarker RS, Yildirim AO, et al. Autoprocessing of neutrophil elastase near its active site reduces the efficiency of natural and synthetic elastase inhibitors. Nat Commun 2015; 6: 6722.

Sly PD, Brennan S, Gangell C, et al. Lung disease at diagnosis in infants with cystic fibrosis detected by newborn screening. Am J Respir Crit Care Med 2009; 180: 146-152. 2013; 368: 1963-1970. MyD88/IRAK/TRAF-6 in human bronchial epithelium. J Biol Chem 2001; 276: 35494-35499.

Fischer BM, Voynow JA. Neutrophil elastase induces MUC5AC gene expression in airway epithelium via a pathway involving reactive oxygen species. Am J Respir Cell Mol Biol 2002; 26: 447-452.

Guyot N, Butler MW, McNally, P, et al. Elafin, an elastase-specific inhibitor, is cleaved by its cognate enzyme neutrophil elastase in sputum from individuals with cystic fibrosis. J Biol Chem 2008; 283: 32377-32385.

Weldon S, McNally P, McElvaney NG, et al. Decreased levels of secretory leucoprotease inhibitor in the Pseudomonas-infected cystic fibrosis lung are due to neutrophil elastase degradation. J Immunol 2009; 183: 8148-8156.

Vandivier RW, Fadok VA, Hoffmann PR, et al. Elastase-mediated phosphatidylserine receptor cleavage impairs apoptotic cell clearance in cystic fibrosis and bronchiectasis. J Clin Invest 2002; 109: 661-670.

Bergsson G, Reeves EP, McNally P, et al. LL-37 complexation with glycosaminoglycans in cystic fibrosis lungs inhibits antimicrobial activity, which can be restored by hypertonic saline. J Immunol 2009; 183: 543-551.

Jackson PL, Xu X, Wilson L, et al. Human neutrophil elastase-mediated cleavage sites of MMP-9 and TIMP-1: implications to cystic fibrosis proteolytic dysfunction. Mol Med 2010; 16: 159-166.

Hartl D, Latzin P, Hordijk P, et al. Cleavage of CXCR1 on neutrophils disables bacterial killing in cystic fibrosis lung disease. Nat Med 2007; 13: 1423-1430.

Sandhaus RA, Turino G. Neutrophil elastase-mediated lung disease. COPD 2013; 10: Suppl 1, 60-63.

Trends Mol Med 2014; 20: 105-115.

Chalmers JD, Hill AT. Mechanisms of immune dysfunction and bacterial persistence in non-cystic fibrosis bronchiectasis. Mol Immunol 2013; 55: 27-34.

Mall M, Grubb BR, Harkema JR, et al. Increased airway epithelial Na+ absorption produces cystic fibrosis-like lung disease in mice. Nat Med 2004; 10: 487-493.

Mall MA, Harkema JR, Trojanek JB, et al. Development of chronic bronchitis and emphysema in beta-epithelial $\mathrm{Na}+$ channel-overexpressing mice. Am J Respir Crit Care Med 2008; 177: 730-742.

Zhou Z, Duerr J, Johannesson B, et al. The ENaC-overexpressing mouse as a model of cystic fibrosis lung disease. J Cyst Fibros 2011; 10: Suppl 2, S172-S182.

Gehrig S, Duerr J, Weitnauer M, et al. Lack of neutrophil elastase reduces inflammation, mucus hypersecretion, and emphysema, but not mucus obstruction, in mice with cystic fibrosis-like lung disease. Am J Respir Crit Care Med 2014; 189: 1082-1092.

Trojanek JB, Cobos-Correa A, Diemer S, et al. Airway mucus obstruction triggers macrophage activation and matrix metalloproteinase 12-dependent emphysema. Am J Respir Cell Mol Biol 2014; 51: 709-720.

Hunninghake GM, Cho MH, Tesfaigzi Y, et al. MMP12, lung function, and COPD in high-risk populations. N Engl J Med 2009; 361: 2599-2608.

Dean RA, Cox JH, Bellac CL, et al. Macrophage-specific metalloelastase (MMP-12) truncates and inactivates ELR+ CXC chemokines and generates CCL2, $-7,-8$, and -13 antagonists: potential role of the macrophage in terminating polymorphonuclear leukocyte influx. Blood 2008; 112: 3455-3464.

Garratt LW, Sutanto EN, Ling KM, et al. Matrix metalloproteinase activation by free neutrophil elastase contributes to bronchiectasis progression in early cystic fibrosis. Eur Respir J 2015; 46: 384-394.

Dreymueller D, Uhlig S, Ludwig A. ADAM-family metalloproteinases in lung inflammation: potential therapeutic targets. Am J Physiol Lung Cell Mol Physiol 2015; 308: L325-L343.

Paulissen G, Rocks N, Gueders MM, et al. Role of ADAM and ADAMTS metalloproteinases in airway diseases. Respir Res 2009; 10: 127.

Saitoh H, Leopold PL, Harvey BG, et al. Emphysema mediated by lung overexpression of ADAM10. Clin Transl Sci 2009; 2: 50-56.

Cooper AM, Hobson PS, Jutton MR, et al. Soluble CD23 controls IgE synthesis and homeostasis in human B cells. J Immunol 2012; 188: 3199-3207.

Van Eerdewegh P, Little RD, Dupuis J, et al. Association of the ADAM33 gene with asthma and bronchial hyperresponsiveness. Nature 2002; 418: 426-430.

Foley SC, Mogas, AK Olivenstein, R, et al. Increased expression of ADAM33 and ADAM8 with disease progression in asthma. J Allergy Clin Immunol 2007; 119: 863-871.

Sahin U, Weskamp G, Kelly K, et al. Distinct roles for ADAM10 and ADAM17 in ectodomain shedding of six EGFR ligands. J Cell Biol 2004; 164: 769-779. 
Inoshima I, Inoshima N, Wilke GA, et al. A Staphylococcus aureus pore
ADAM10 to cause lethal infection in mice. Nat Med 2011; 17: 1310-1314.

61 Greene CM, McElvaney NG. Proteases and antiproteases in chronic neutrophilic lung disease - relevance to drug discovery. Br J Pharmacol 2009; 158: 1048-1058.

62 Hiemstra PS. Novel roles of protease inhibitors in infection and inflammation. Biochem Soc Trans 2002; 30: 116-120.

63 Meyer M, Jaspers I. Respiratory protease/antiprotease balance determines susceptibility to viral infection and can be modified by nutritional antioxidants. Am J Physiol Lung Cell Mol Physiol 2015; 308: L1189-L1201.

64 Janciauskiene SM, Bals R, Koczulla R, et al. The discovery of alpha1-antitrypsin and its role in health and disease. Respir Med 2011; 105: 1129-1139.

65 Roussel BD, Irving JA, Ekeowa UI, et al. Unravelling the twists and turns of the serpinopathies. FEBS J 2011; 278: 3859-3867.

66 Gooptu B, Lomas DA. Polymers and inflammation: disease mechanisms of the serpinopathies. J Exp Med 2008; 205: 1529-1534.

67 Davies MJ, Lomas DA. The molecular aetiology of the serpinopathies. Int J Biochem Cell Biol 2008; 40: 1273-1286.

68 Gooptu B, Dickens JA, Lomas DA. The molecular and cellular pathology of alpha(1)-antitrypsin deficiency. Trends Mol Med 2014; 20: 116-127.

69 Alam S, Li Z, Janciauskiene S, et al. Oxidation of Z alpha1-antitrypsin by cigarette smoke induces polymerization: a novel mechanism of early-onset emphysema. Am J Respir Cell Mol Biol 2011; 45: $261-269$.

70 Alam S, Li Z, Atkinson C, et al. Z alpha1-antitrypsin confers a proinflammatory phenotype that contributes to chronic obstructive pulmonary disease. Am J Respir Crit Care Med 2014; 189: 909-931.

71 Lior Y, Geyra A, Lewis EC. Therapeutic compositions and uses of alpha1-antitrypsin: a patent review (2012-2015). Expert Opin Ther Pat 2016; 26: 581-589.

72 Pemberton PA, Kobayashi D, Wilk BJ, et al. Inhaled recombinant alpha 1-antitrypsin ameliorates cigarette smoke-induced emphysema in the mouse. COPD 2006; 3: 101-108.

73 Griese M, Latzin P, Kappler M, et al. alpha1-Antitrypsin inhalation reduces airway inflammation in cystic fibrosis patients. Eur Respir J 2007; 29: 240-250.

74 McElvaney NG, Doujaiji B, Moan MJ, et al. Pharmacokinetics of recombinant secretory leukoprotease inhibitor aerosolized to normals and individuals with cystic fibrosis. Am Rev Respir Dis 1993; 148: 1056-1060.

75 auf dem Keller U, Prudova A, Eckhard U, et al. Systems-level analysis of proteolytic events in increased vascular permeability and complement activation in skin inflammation. Sci Signal 2013; 6: rs2.

76 Rodriguez D, Morrison CJ, Overall CM. Matrix metalloproteinases: what do they not do? New substrates and biological roles identified by murine models and proteomics. Biochim Biophys Acta 2010; 1803: 39-54.

77 Dean RA, Overall CM. Proteomics discovery of metalloproteinase substrates in the cellular context by iTRAQ labeling reveals a diverse MMP-2 substrate degradome. Molecular \& cellular proteomics: MCP 2007; 6: 611-623.

78 Tam EM, Morrison, CJ Wu, YI, et al. Membrane protease proteomics: Isotope-coded affinity tag MS identification of undescribed MT1-matrix metalloproteinase substrates. Proc Natl Acad Sci USA 2004; 101: 6917-6922.

79 Fortelny N, Cox JH, Kappelhoff R, et al. Network analyses reveal pervasive functional regulation between proteases in the human protease web. PLoS Biol 2014; 12: e1001869.

80 Bellac CL, Dufour A, Krisinger MJ, et al. Macrophage matrix metalloproteinase-12 dampens inflammation and neutrophil influx in arthritis. Cell Rep 2014; 9: 618-632.

81 Lange PF, Huesgen PF, Overall CM. TopFIND 2.0--linking protein termini with proteolytic processing and modifications altering protein function. Nucleic Acids Res 2012; 40: D351-D361.

82 Lange PF, Overall CM. TopFIND, a knowledgebase linking protein termini with function. Nat Methods 2011; 8: 703-704.

83 Butler GS, Overall CM. Proteomic identification of multitasking proteins in unexpected locations complicates drug targeting. Nat Rev Drug Discov 2009; 8: 935-948.

84 Overall CM, Kleifeld O. Tumour microenvironment - opinion: validating matrix metalloproteinases as drug targets and anti-targets for cancer therapy. Nat Rev Cancer 2006; 6: 227-239.

85 Overall CM, Lopez-Otin, C. Strategies for MMP inhibition in cancer: innovations for the post-trial era. Nat Rev Cancer 2002; 2: 657-672.

86 Cobos-Correa A, Trojanek, JB Diemer, S, et al. Membrane-bound FRET probe visualizes MMP12 activity in pulmonary inflammation. Nat Chem Biol 2009; 5: 628-630.

87 Gehrig S, Mall MA, Schultz C. Spatially resolved monitoring of neutrophil elastase activity with ratiometric fluorescent reporters. Angew Chem Int Ed Engl 2012; 51: 6258-6261.

88 Withana NP, Ma X, McGuire HM, et al. Non-invasive imaging of idiopathic pulmonary fibrosis using cathepsin protease probes. Sci Rep 2016; 6: 19755.

89 Giannandrea M, Parks WC. Diverse functions of matrix metalloproteinases during fibrosis. Dis Model Mech 2014; 7: 193-203.

90 Pardo A, Cabrera S, Maldonado M, et al. Role of matrix metalloproteinases in the pathogenesis of idiopathic pulmonary fibrosis. Respir Res 2016; 17: 23.

91 Lu P, Weaver VM, Werb Z. The extracellular matrix: a dynamic niche in cancer progression. J Cell Biol 2012; 196: 395-406

92 Lee H, Kim YP. Fluorescent and bioluminescent nanoprobes for in vitro and in vivo detection of matrix metalloproteinase activity. BMB Rep 2015; 48: 313-318.

93 Ruan S, He Q, Gao H. Matrix metalloproteinase triggered size-shrinkable gelatin-gold fabricated nanoparticles for tumor microenvironment sensitive penetration and diagnosis of glioma. Nanoscale 2015; 7: 9487-9496.

94 Tauro M, McGuire J, Lynch CC. New approaches to selectively target cancer-associated matrix metalloproteinase activity. Cancer Metastasis Rev 2014; 33: 1043-1057.

95 Wang Y, Lin T, Zhang W, et al. A prodrug-type, MMP-2-targeting nanoprobe for tumor detection and imaging. Theranostics 2015; 5: 787-795. 
van Rijt SH, Bein T, Meiners S. Medical nanoparticles for next generation drug delivery to the lungs. Eur Respir J 2014; 44: 765-774

97 van Rijt SH, Bölükbas DA, Argyo C, et al. Protease-mediated release of chemotherapeutics from mesoporous silica nanoparticles to ex vivo human and mouse lung tumors. ACS Nano 2015; 9: 2377-2389.

98 Naumnik W, Niklińska W, Ossolińska M, et al. Serum cathepsin K and cystatin C concentration in patients with advanced non-small-cell lung cancer during chemotherapy. Folia Histochem Cytobiol 2009; 47: $207-213$.

99 Chapman HA, Riese RJ, Shi GP. Emerging roles for cysteine proteases in human biology. Annu Rev Physiol 1997; 59: $63-88$.

100 Huang HB, Peng XN, Nakajima J. Advances in the study of biomarkers of idiopathic pulmonary fibrosis in Japan. Biosci Trends 2013; 7: 172-177.

101 Rao NV, Wehner, NG Marshall, BC, et al. Characterization of proteinase-3 (PR-3), a neutrophil serine proteinase. Structural and functional properties. J Biol Chem 1991; 266: 9540-9548.

102 Müllberg J, Durie FH, Otten-Evans C, et al. A metalloprotease inhibitor blocks shedding of the IL-6 receptor and the p60 TNF receptor. J Immunol 1995; 155: 5198-5205.

103 Moss ML, Bomar M, Liu Q, et al. The ADAM10 prodomain is a specific inhibitor of ADAM10 proteolytic activity and inhibits cellular shedding events. J Biol Chem 2007; 282: 35712-35721.

104 Zou J, Zhang R, Zhu F, et al. ADAM33 enzyme properties and substrate specificity. Biochemistry 2005; 44: 4247-4256.

105 Tiede SL, Wassenberg M, Christ K, et al. Biomarkers of tissue remodeling predict survival in patients with pulmonary hypertension. Int J Cardiol 2016; 223: 821-826.

106 Moss ML, Rasmussen FH. Fluorescent substrates for the proteinases ADAM17, ADAM10, ADAM8, and ADAM12 useful for high-throughput inhibitor screening. Anal Biochem 2007; 366: 144-148.

107 Sokai A, Handa T, Tanizawa K, et al. Matrix metalloproteinase-10: a novel biomarker for idiopathic pulmonary fibrosis. Respir Res 2015; 16: 120.

108 Vandooren J, Van den Steen PE, Opdenakker G. Biochemistry and molecular biology of gelatinase B or matrix metalloproteinase-9 (MMP-9): the next decade. Crit Rev Biochem Mol Biol 2013; 48: 222-272. 